\title{
Research on Detection Technology of Corona Discharge Fault in Power Equipment
}

\author{
Huang Hua ${ }^{1}$, Liu Fangwu², Yuan Shidong ${ }^{2}$, Xiao Rong ${ }^{1}$, and Ding Kun ${ }^{2, *}$ \\ 1State Grid Shanghai Electric Power Research Institute, Shanghai 200437, China \\ 2Shanghai Institute of Technical and Physics, CAS, Shanghai 200083, China \\ ${ }^{*}$ Corresponding author
}

\begin{abstract}
Key words: power equipment failure; ultraviolet; infrared; image fusion
Abstract: UV corona on power system can reflect the location of the fault and the severity of the fault, the traditional UV and infrared detection equipment can only use the band and the visible light band image of the power system fault detection. In this paper, a power system fault detection system based on ultraviolet and infrared dual-band images is designed. The principle of UV imaging detection and image fusion are introduced respectively. The software of the host computer is written by MFC. The software can acquire both ultraviolet and infrared, The two images are fused using the image fusion algorithm based on edge detection and cross correlation and the highest point temperature is plotted. The field measurement shows that the system can detect the failure of the power equipment in time, and has a good practical value, which provides an efficient detection solution for the fault detection of the power equipment.
\end{abstract}

\section{Introduction}

Power equipment in the long-term outdoor high temperature, high pressure and full load, it is prone to failure, and even the formation of accidents, so the need for regular testing of the state of power equipment. Early maintenance of the electrical equipment is a fault after the repair, this way the sudden failure will often cause huge losses. The daily preventive maintenance of electrical equipment can effectively reduce and prevent the occurrence of electrical equipment accidents, has become the power sector system. The traditional preventive maintenance must be in the case of power failure to be detected, the state after the shutdown device is often not the same as the run-time, affecting the detection accuracy and accuracy. Therefore, the uninterrupted power equipment, real-time online detection is an urgent need to solve the problem ${ }^{[1]}$ 。

The spectral range of the ultraviolet band is 10 400 $\mathrm{nm}$. The sun also contains the spectral information of the ultraviolet band, Where the following spectrum $280 \mathrm{~nm}$ passes through the atmosphere, almost completely absorbed, and thus known as the UV-solar blind band. When the partial discharge electrical device capable of emitting ultraviolet light in the UV-solar blind band, So corona discharge detection can be achieved by detecting the spectrum of the UV-solar blind band ${ }^{[2]}$.By performing infrared imaging of the discharge point, the thermal status of the device can be obtained and the state of the power equipment can be judged according to the characteristic and the correlation criterion ${ }^{[3,4]}$.

In this paper, a corona discharge fault detection system is designed to collect the ultraviolet and infrared images of the fault point in real time. The algorithm can be used to register and fuse the image, and the corona position and the temperature of the highest temperature in the region can be obtained. This technology provides an efficient detection scheme for on-line detection of corona discharge.

\section{System principle}

\subsection{UV imaging detection module system principle}

The ICCD is mainly composed of a photocathode, a microchannel plate (MCP) amplifier, a phosphor screen, an optical fiber cone and a visible light CCD camera. The ICCD is composed of an ultraviolet 
sensor module light source issued by the ultraviolet band signal filter, the radiation to the UV-sensitive photoelectric cathode, the photoelectric conversion generated by the photoelectron by high-voltage electric field acceleration, through the MCP for electron multiplication, multiplied after the electronic bombardment of the screen, to achieve Photoelectron to photon conversion, the photon passes through the fiber optic cone to reach the visible CCD camera, and finally obtains the UV image of the fault target. The structure of the UV ICCD camera is shown in Fig $1^{[5]}$ 。

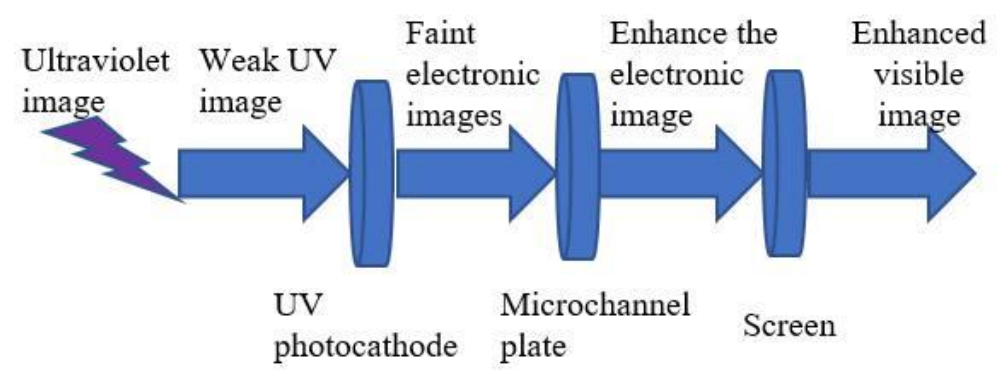

Fig.1 ICCD structure diagram

In a certain range, the gain and detection accuracy of a linear relationship, by adjusting the ICCD gain can improve the detection sensitivity ${ }^{[6]}$, get the best test results.

\subsection{Image Fusion Technology Based on Geometric Relation}

The system uses ultraviolet and infrared cameras to obtain two bands of images, according to the industry commonly used design standards, UV camera field angle is $8^{\circ} \times 6^{\circ}$, the angle of view of the infrared camera is $25^{\circ} \times 18^{\circ}$. The angle of view of the two cameras is different, the geometric size of a single pixel is also different, so the fusion of two bands of the image, we need to register a single pixel to normal display of the fault point of information. The system uses an image registration algorithm based on geometric relations to register two images ${ }^{[7,8]}$.As the UV camera field angle is smaller than the infrared camera, so get a single UV image smaller than the infrared image. We define the positional relationship between the UV image and the infrared image by geometric reduction and registration tests, and superimpose the ultraviolet image into the center of the infrared image.

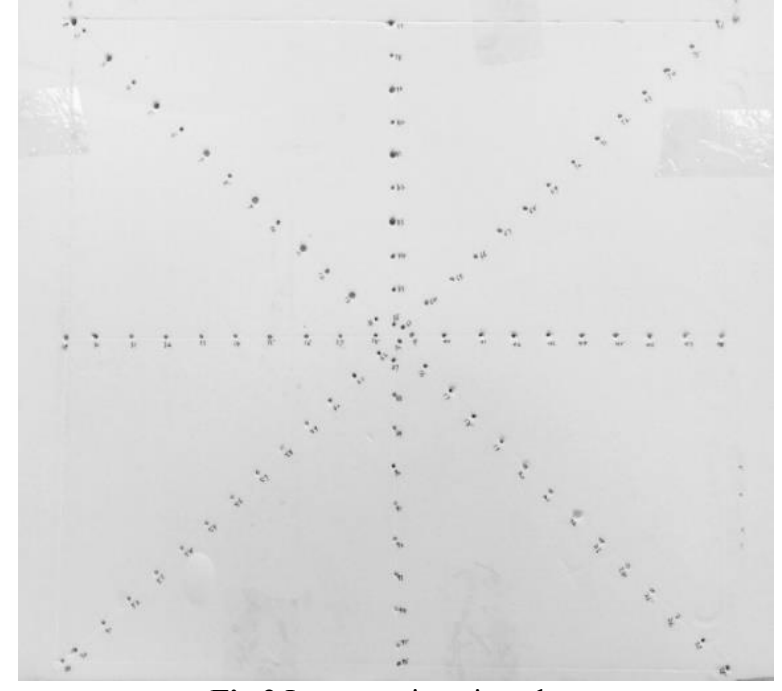

Fig.2 Image registration plate

The specific image registration fusion steps are as follows:

(1) the production of $1000 \times 1000 \mathrm{~mm}$ calibration plate as shown in Figure 2, and in the horizontal, vertical, diagonal four directions equidistant processing 80 diameter of $5 \mathrm{~mm}$ hole.

(2) Using the fluorescent lamp evenly after each circular hole of the calibration plate, each of the 80 pieces of the image to be registered and the reference image was obtained using the ultraviolet infrared spectroscopic camera, respectively. 
(3) Obtain the edge information of each imaging point in the image to be registered by the ellipse and the least squares method to obtain the respective central points of each ellipse and calculate the amount of translation with the corresponding infrared band reference image.

Due to the position of the camera lens and the calibration plate used, all the circles in the calibration plate are elliptical in the UV image, and the coordinates of the ellipse center point can represent the position of each ellipse in the image. Elliptic general equation formula:

$$
A x^{2}+B x y+C y^{2}+D x+E y+F=0
$$

In the two-dimensional UV image, the edge information of the ellipse is $n$, and the $n$ points are minimally multiplied to obtain the coefficients in the equation. The central coordinates of the ellipse are:

$$
\left\{\begin{array}{l}
x_{0}=\frac{B E-2 C D}{4 A C-B^{2}} \\
y_{0}=\frac{B D-2 A E}{4 A C-B^{2}}
\end{array}\right.
$$

The required coefficients are taken into the formula to obtain the coordinates of the central point of the ellipse, which corresponds to the point in the infrared field of view. The least squares method is used to obtain the final translational value. The obtained 80 pairs of corresponding coordinates are fitted by least squares method. The obtained linear coefficient is the translational amount of UV and infrared corresponding points.

(4) the use of four corners in the image of the location, calculate the infrared and ultraviolet image scaling;

(5) the UV image and infrared images were fused after the axial, horizontal, diagonal four directions of the reference curve, and the calibration plate geometry compared to calculate the camera system aberration, usually in the lens distortion in smaller cases the reference value can be ignored.

Using the above algorithm, will complete the UV image and infrared image registration and fusion.

\section{System development and calibration experiments}

\subsection{System development}

The response range of the infrared camera is $8 \sim 14 \mu m$, through the high-speed data transmission interface will be collected to the infrared image transmission to the host computer, the host computer through the RS232 interface to send control commands to the infrared camera, used to achieve image contrast, brightness and integration time adjustment. The upper computer receives the infrared image and processes the image. The infrared image is subjected to pseudo color processing according to the pre-calibrated temperature value, and the highest temperature point is identified.

The project uses a sun blind UV camera, the detection band is $240 \sim 280 \mathrm{~nm}$, through high-speed data transmission interface and the host computer for image transmission, UV images for the gray scale display.

When the system is designed, the optical path of the two cameras is strictly fixed on the horizontal optical axis so that the image fusion algorithm can fuse the image through the feature point localization and the edge region detection.

Figure 3 is the system block diagram.

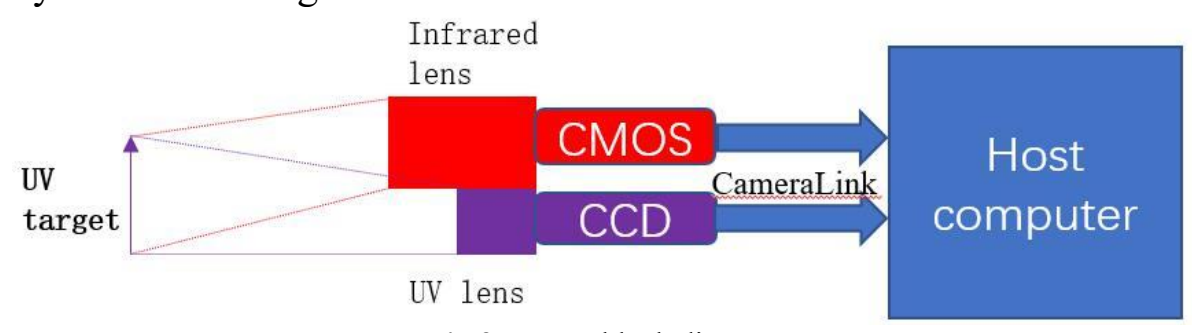

Fig.3 System block diagram 
The host computer software using MFC prepared, the system boot, the UV camera and infrared cameras were obtained from the relevant band images, the Camera Link interface to the host computer, the host computer software in accordance with the above image registration algorithm for registration and integration, real-time display the target region of the double spectral image, and according to the need for a single spectrum of images and fusion images stored in the specified location.

\subsection{System sensitivity calibration experiment}

After the development of the equipment, the UV band sensitivity calibration experiment was carried out. The experimental system was shown in Fig.4, Zolix Omni- $\lambda 300$ monochromator was used as the UV light source, and the monochromatic light of the wavelength range of $240 \mathrm{~nm} \sim 280 \mathrm{~nm}$ was output. The step wavelength was $10 \mathrm{~nm}$. The self-developed corona discharge fault detection system was used with the OFIL UV detector. UV light source for observation.

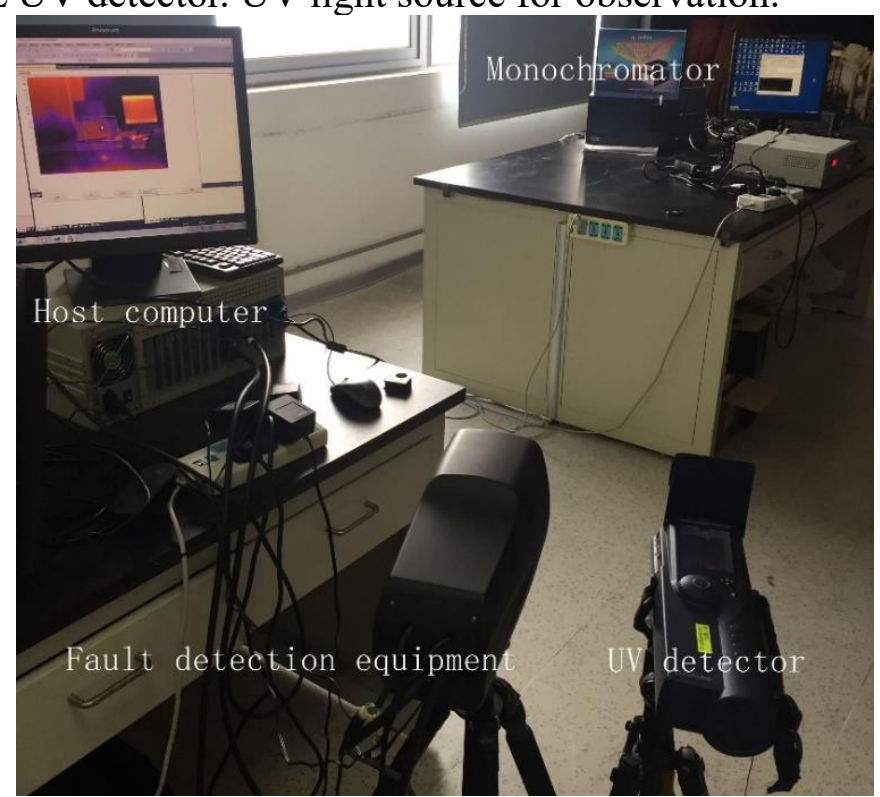

Fig.4 UV band sensitivity calibration experiment 

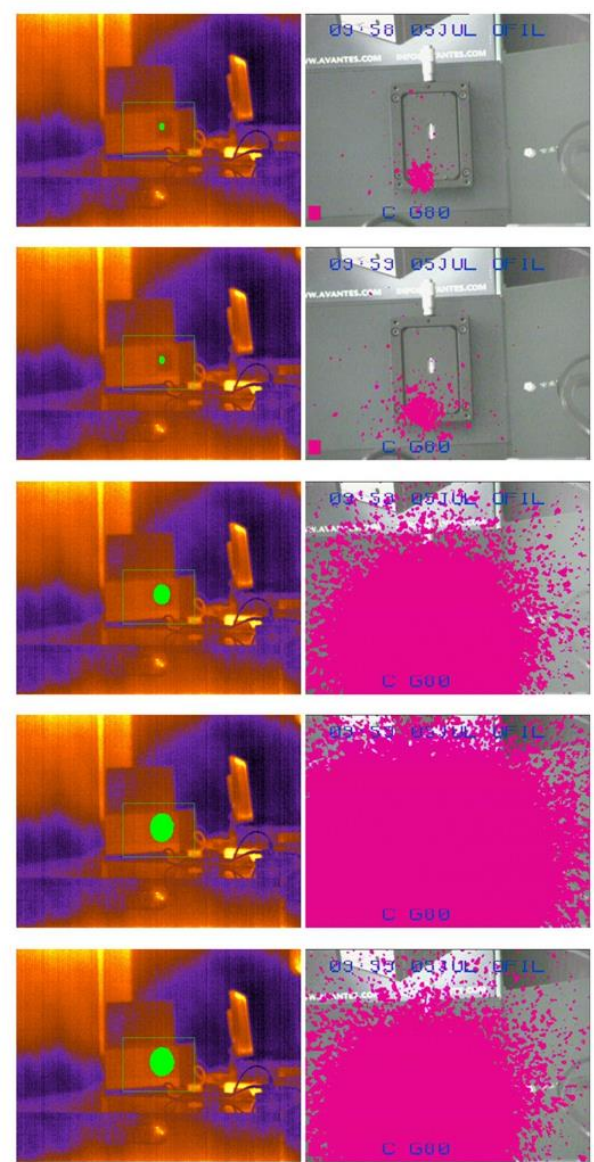

Fig.5 UV band sensitivity calibration experiment result

From figure 5 , it can be seen that in the $240 \mathrm{~nm} \sim 280 \mathrm{~nm}$ band, our equipment can obtain the blind ultraviolet band image, and the self-study device can obtain the same value as the gain of the UV detector. The observed trend of photon number is the same as the change of photon number observed by UV detector.

\section{Summary}

This paper describes the design of a device for the power equipment of charged solar blind ultraviolet and infrared imaging detection based on solar blind UV can simultaneously obtain the power equipment and the infrared image, the image registration algorithm for registration of the geometric relationship between the two band image fusion based on the experimental results show that this device can achieve the expected design the target, and has good practical value, provides an efficient solution for real-time online detection of corona discharge fault of power equipment.

\section{References}

[1] WU Li-gang,HE Wen-rong,Hu Jin-sun, et al.Study on Daytime Camera for Corona Discharge Detection[J]. SEMICONDUCTOR OPTOELECTRONICS, 2010, (03): 443-446+50.

[2] CU Hao-yang,CHEN Lin,TANG Nai-yun,et al. The Application of UV Imagery Technology in Electrical Equipment PD Detection[J]. Journal of Shanghai University of Electric Power, 2012, (01): 93-96.

[3] BIAN Yu-ping, KANG Yu-bin. Application of IR and UV detection technology in UHV transmission lines[J].North China Electric Power, 2012(2): 23-26.

[4] KUANG Lei,GU Yan. Research on the Application of UV Image Intensifier in Security Detection of Power Equipment.[J]. Infrared Technology, 2015, (11): 986-990. 
[5] DONG Xiao-qing, WU Jun,WANG Hao, et al. Research on airborne ultraviolet equipment and fault diagnosis technique for transmission line[J]. JOURNAL OF EIECTRIC POWER SCIENCE AND TECHNOLOGY, 2017, 01): 117-122.

[6] ZHAO Yu-huan,YAN Feng,ZHOU Yue,et al. Radiometric calibration of ultraviolet ICCD detector[J].Optics and Precision Engineering, 2008, (09): 1572-1576.

[7] CHEN Liang,ZHOU Meng-zhe,CHEN He.A Method for Image Registration Combined by Edge Region and Cross Correlation[J]. Transactions of Beijing Institute of Technology, 2016, (03): 320-325.

[8] JIN Li-jun,ZHANG Da,DUAN Shao-Hui,et al. Recognition of Contamination Grades of Insulators Based on IR and UV Image Information Fusion[J]. TRANSACTIONS OF CHINA ELECTROTECHNICAL SOCIETY, 2014, (08): 309-318. 